\title{
Suitability of a SiPM Photodetector for Implementation in an Automated Thermoluminescent Dosimeter Reader
}

\author{
M.P. Taggarta, ${ }^{\mathrm{a}, *}$, A. Charoupa ${ }^{\mathrm{a}}$, M.W.J. Hubbard ${ }^{\mathrm{a}}$, S.M. Jafari ${ }^{\mathrm{a}, \mathrm{b}, \mathrm{c}}$, A. Lohstroh ${ }^{\mathrm{a}}$, M. Stroud ${ }^{\mathrm{c}}$ \\ ${ }^{a}$ Department of Physics, University of Surrey, Guildford, Surrey, GU2 7XH, U.K. \\ ${ }^{b}$ Radiotherapy Physics, Level B, Queen Alexandra Hospital, Portsmouth, PO6 3LY, U.K. \\ 'TRUEinvivo, The Surrey Technology Centre, Surrey Research Park, Guildford, Surrey, GU2 7YG, UK
}

\begin{abstract}
It is widely known that SiPM photodetectors have a strong temperature dependence. TrueInvivo is currently developing an automated TLD reader with the intention of replacing the PMT with a SiPM, taking advantage of the low power consumption, small form-factor, and low cost the SiPM has to offer. Here we discuss our initial investigations into the suitability of this switch in applied technology, present spectroscopic response as a function of SiPM temperature, and the effectiveness of some options to mitigate this concern. It was found that while the SiPM is indeed affected by variations in temperature a recovery of $\sim 50-60 \%$ in gain and resolution could be achieved with a rudimentary cooling system.
\end{abstract}

Keywords: glass bead TLD, thermoluminescence, silicon photomultiplier

\section{Introduction}

The silicon photomultiplier ( $\mathrm{SiPM})$ is becoming increasingly popular in a variety of fields, from nuclear security [1, 2], to radiopharmeceutical quality control [3], to health physics [4], to medical imaging [5, 6], to name but a few. Effectively, any field that was formerly the domain of the photomultiplier tube is now rapidly being invaded with solid state technology. Over recent years great strides have been made in the reduction of the SiPM dark count allowing for their use in applications where the activity is expected to be very low, and the typical gain of $10^{6}$ is comparable to, if not greater than, that of many common photomultiplier tubes (PMT)[7--10]. Coupled to the small form-factor of the devices and the fact that they typically require low operating voltages, SiPMs are a very promising prospect for many low light-level detection ap-

\footnotetext{
*Corresponding author

Email address: m.taggart@surrey . ac .uk (M.P. Taggart)
}

plications. In particular their influx in the world of medical physics is opening up new realms of study and research opportunity, with the intrinsic magnetic insensitivity of solid state devices compared to a PMT utilising stepped electric potentials for amplification, the implementation of SiPM devices allows for the use of PET-MRI systems to become more widespread[11-[13].

In parallel to technical developments in the world of photodetection, since 2007 the University of Surrey has been at the forefront of research into micro-silica dosimetry[14]. There has been an increased demand for dosimetry on a micro-scale to compliment and allow thorough exploitation of the comparable advancements in radiotherapy procedures. Treatment techniques such as Cyberknife[15], intensity-modulated radiation therapy (IMRT) [16, 17], and stereotactic body radiation therapy (SBRT) [18] rely upon high dose and dose gradients, high spatial resolution, and high linearity[19-21]. The glass beads described initially in [22] have been shown to have 
a linear dose response over a wide range with exceptional reproducibility. Furthermore, the read-out and analysis of the glass beads is not dissimilar to that used for typical standard thermoluminescent dosimeters (TLDs), ie. placed onto a planchett and subjected to contact heating up to $400^{\circ} \mathrm{C}[10,23,25]$. Indeed, the performance of the beads has previously been investigated using a Toledo 654[22, 26, 27] and a Risø DA-20[28-30], both machines widely used for dosimetric auditing with materials such as $\mathrm{LiF}: \mathrm{Mg}, \mathrm{Cu}, \mathrm{Ph}$ [31, 32] and $\mathrm{Al}_{2} \mathrm{O}_{3}: \mathrm{C}[33,34]$.

Although SiPMs are becoming common within Medical Physics due to their implementation in PET-MRI, for applications such as TLD or OSLD (Optically Stimulated Luminescence Dosimetry) the temperature dependence typical of most solid state devices presents an additional engineering challenge[35-38] simply due to the proximity of the contact heater to the photosensor. The breakdown voltage $\left(\mathrm{V}_{b r}\right)$ of the SiPM is one of the key operating parameters, described as the voltage at which a semiconductor ceases to act as an insulator[10, 39]. The breakdown voltage is linearly correlated to the temperature and affects both the gain and dark current of the SiPM, with the gain in particular being reduced significantly when temperature is increased, as the increase in $\mathrm{V}_{b r}$ naturally reduces the gain-determining overvoltage (difference between the operating/supply and breakdown voltages) of the system where the supply voltage remains constant. Typically this temperature dependence is compensated for by one (or more) of a number of approaches; gain-matching[40] is an effective approach, albeit relatively simplistic, whilst active methods such as immersion [41] or thermoelectric pumping [42] have also been exploited.

Here we investigate whether the silicon photomultiplier is a technology that can be applied to the next generation of thermoluminescent dosimeter readers.

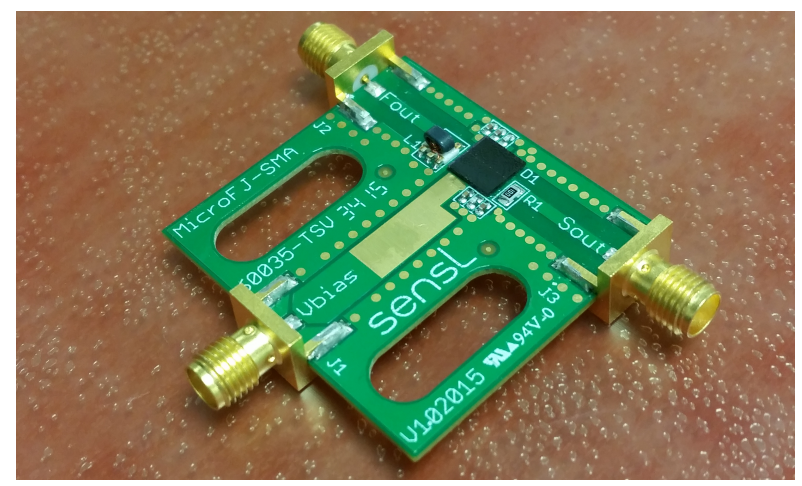

Figure 1: A SensL MicroFJ-SMA-60035 SiPM.

\section{Experimental Setup}

All experiments were performed on SensL's latest model SiPM, the J-series, to take advantage of the high photodetection efficiency (PDE) and low dark count. The Micro-FJ-60035-TSV SiPM is shown in Figure 1] Table 1 lists some of the important characteristics of the studied SiPM, however of most relevance to this work is the variation in breakdown voltage given as $21.5 \mathrm{mV} /{ }^{\circ} \mathrm{C}[43]$.

Table 1: Some key operating characteristics of the SensL J-series SiPM[43]. Note that the PDE does not contain afterpulsing or crosstalk.

\begin{tabular}{c|c|c}
\hline & Amount & Unit \\
\hline Active area & $6.07 \times 6.07$ & $\mathrm{~mm}^{2}$ \\
$\mathrm{~V}_{b r}$ & 24.7 & $\mathrm{~V}$ \\
Microcell size & 35 & $\mu \mathrm{m}$ \\
No. of microcells & 22292 & \\
Peak PDE & 38 & $\%$ \\
Dark count & 50 & $\mathrm{kHz} / \mathrm{mm}^{2}$ \\
Gain & 2.9 & $\times 10^{6}$ \\
Recovery & 50 & $\mathrm{~ns}$ \\
\hline
\end{tabular}

The temperature sensitivity of the SiPM is investigated by thermally coupling the device to a heating system depicted in Figure 2 all contained within a light-tight metal box. Thermally coupling the SiPM to a ceramic plate housing a PT100 resistance temperature detector allows for continuous monitoring of the SiPM temperature. A preliminary study on an equivalent piece of $1.2 \mathrm{~mm}$ 


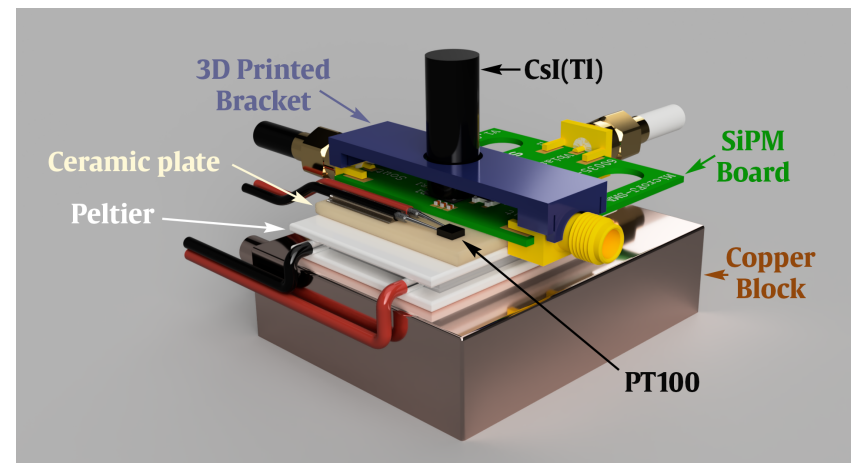

Figure 2: Rendering of the experimental set-up for the direct heating portion of this work. For investigations into the spectroscopic response a cylindrical piece of $\mathrm{CsI}(\mathrm{Tl})$ is optically coupled to the SiPM, otherwise both it and the supporting 3D-printed bracket are removed.

thick FR4 PCB to replicate any dampening effect caused by the SiPM evaluation board determined that thermal equilibrium was reached after $\sim 1$ minute, although as a precaution at least 5 minutes stabilisation time was allowed prior to each measurement. The PT100 layer is subsequently coupled with Electrolube silicone heat transfer compound to a CP1.0-31-05L Peltier module which itself is coupled to a copper heat-sink with a pumped water flow from an RTE-2000. As described in [42], the Peltier thermoelectric module operates via the Peltier effect to facilitate a heat differential across the device, thereby creating a "hot" and "cold" side. The device can then act either as a "heater" or a "cooler" to the SiPM depending on its orientation. Furthermore, the addition of the RTE-2000 to the heating chain allows for a greater degree of temperatures to be investigated, with coarse temperature set with the water flow before the Peltier is used for fine tuning.

\subsection{Measurements}

The key operating parameter of a SiPM is the breakdown voltage $\left(\mathrm{V}_{b r}\right)$, described as the voltage at which a semiconductor ceases to act as an insulator[10, 39]. This was determined for the SensL device studied using a Keithley 487 picoammeter. The characteristic plot of current against voltage (an IV curve) is shown in Figure 3 for ambient temperature. In common with the SensL guide-

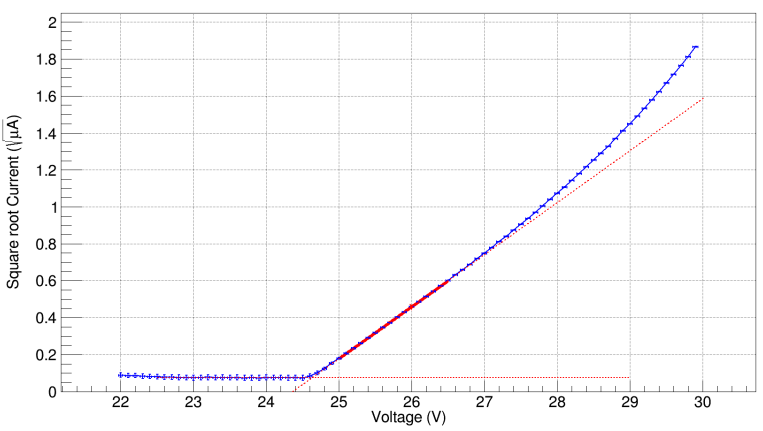

Figure 3: The IV curve of the SiPM at ambient conditions. The breakdown voltage is determined from the straight line fit.

lines the breakdown voltage is defined as "the value of the voltage intercept of a straight line fit to a plot of the square root of the current versus over-voltage" [43].

\section{2. $\gamma$ Spectroscopy}

The spectroscopic response of the SiPM as a function of temperature was investigated using the same system described previously, but with the addition of a scintillator optically coupled to the SiPM. The scintillator selected was a cylinder of thallium-doped caesium iodide, or $\mathrm{CsI}(\mathrm{Tl})$, measuring $20 \mathrm{~mm}$ in length and with a $7 \mathrm{~mm}$ diameter. One face was optically coupled to the SiPM with the remainder tightly wrapped in reflective foil to improve light collection. CsI(Tl) is a well-studied scintillator that has found uses in a variety of applications from medical physics [44-48] to more fundamental research [49-51]. It was selected due to its high stopping power, which increases with both density and effective atomic number $\left(\rho=4.51 \mathrm{~g} / \mathrm{cm}^{3}, Z_{\text {eff }}=54\right)$, very good light yield $(54,000$ photons $/ \mathrm{MeV})$, and it being the only scintillator in the laboratory that would physically fit on the space allowed by the SiPM evaluation board. As can be seen in Figure $4 . \mathrm{CsI}(\mathrm{Tl})$ has a peak wavelength at around $550 \mathrm{~nm}$, somewhat offset from the peak responsivity of the SiPM at $420 \mathrm{~nm}$, however the high light yield compensates for this mismatch.

The output of the SiPM was taken to an Ortec 


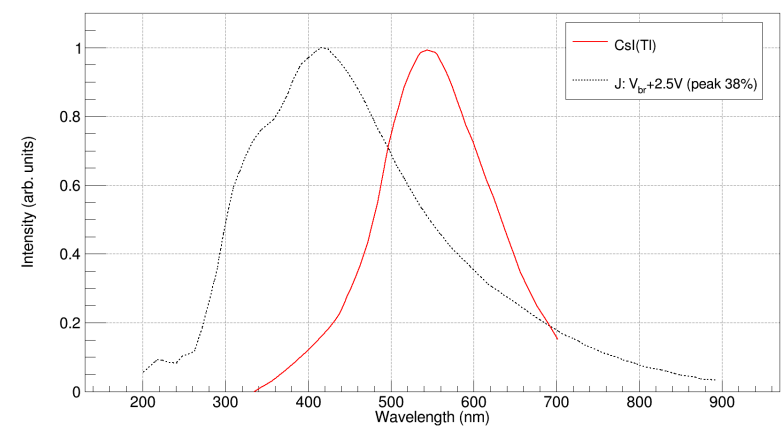

Figure 4: Overlay of the photodetection efficiency of the J-series SiPM with the relative emmision with respect to wavelength of the CsI(Tl) scintillator. Data extracted from [43, 52].

570 spectroscopy amplifier for acquisition by an Ortec EasyMCA 8k channel multichannel analyzer. The MCA has a 0-10 V input dynamic range and the conversion gain was set to 2048 channels due to its use with the CsI(Tl) scintillator via the Maestro control software[53]. All data is post-processed using a ROOT analysis code to produce the $\gamma$ spectra. Standard laboratory $\gamma$ ray sources, ${ }^{137} \mathrm{Cs}$ $(107 \mathrm{kBq})$ and ${ }^{22} \mathrm{Na}(190 \mathrm{kBq})$ were investigated.

\subsection{Proximity Heating}

The Peltier technique has two major drawbacks. The first is that this is not an accurate representation of the conditions a SiPM would be subjected to when enclosed within a TLD reader, there will be limited thermal conduction and heat transfer would be primarily through radiation. The second is the temperature range available for investigation, primarily that the temperatures required to empty traps within the glass beads are sufficient to liquify the solder used in the evaluation board. However, that the first drawback exists means that the second drawback might be mitigated.

If the Risø DA-20 TLD reader is used as an example for a standard TLD heating system[28], the heating element lifts the sample into position under the photosensor, ramps the temperature of the planchett up to around $400^{\circ} \mathrm{C}$ (depending on the TLD material), before lowering the sample again away from the sensor. Additionally, the
Risø has a nitrogen supply which can prevent oxidation on the heating element at high temperatures.

To simulate the heat exposure during TLD readout a ZENY 937D+ soldering iron was placed close to the SiPM at an approximately $2 \mathrm{~mm}$ stand-off. The iron was oriented that the flat edge bisected the SiPM face and was secured $\sim 2 \mathrm{~mm}$ away. The iron was then held at increasingly high temperatures and IV measurements were repeatedly taken to investigate the stability of the SiPM over prolonged high temperature exposure.

\subsection{Active Cooling}

The Peltier module acts as a heat pump, moving heat from one side to the other. In one orientation, that described thus far, it provides a heat source, however, by reversing the module it acts to cool the surface it is in contact with. In this case the heat in the SiPM is actively removed by the Peltier and water cooling of the RTE-2000. In the reverse orientation, that of the cool side towards the SiPM, the Peltier is used to reduce any external heating effect due to the nearby soldering iron and return the value of the breakdown voltage back towards that recorded under room temperature conditions and therefore potentially boosting the gain.

\section{Results and Discussion}

The baseline IV measurement establishing the breakdown voltage of the device was shown in Figure 3 establishing the value of $\mathrm{V}_{b r}$ as $24.62 \pm 1.75 \mathrm{~V}$ at room temperature. This is in common with the typical values quoted by the manufacturer [43] and measured by the authors for similar units[54].

The initial direct heating measurements took the SiPM beyond its rated temperature range of $-40^{\circ} \mathrm{C}-85^{\circ} \mathrm{C}$ [43] with the highest temperature at which data was recorded for being $154^{\circ} \mathrm{C}$, the maximum provided by the boosted (ie. with $90^{\circ} \mathrm{C}$ water pumped through the heat sink) 


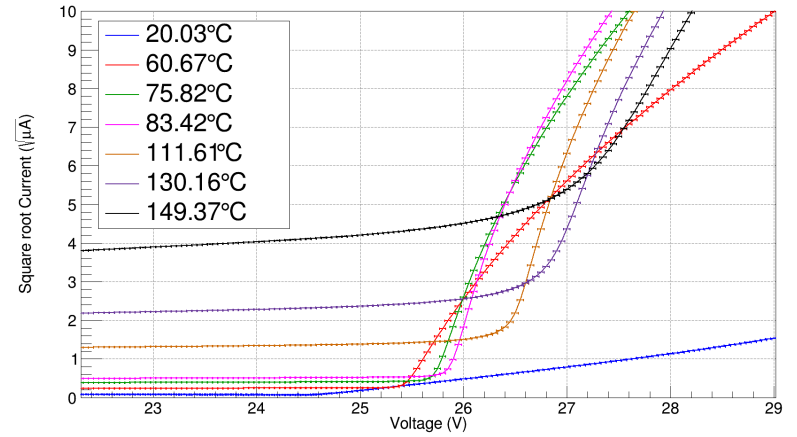

Figure 5: Some example IV curves with the SiPM heated via the direct conduction method.

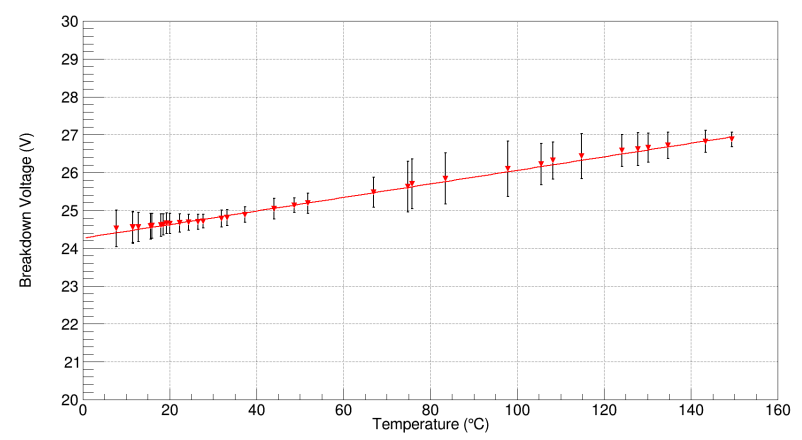

Figure 6: Breakdown Voltage as a function of SiPM temperature.

Peltier. A sample set of IV curves is depicted in Figure 5 which shows that as temperature of the device is increased, so does the value of the breakdown voltage. Within the rated temperature range the value of $\mathrm{V}_{b r}$ increases from $24.67 \pm 2.16 \mathrm{~V}$ at $20.03^{\circ} \mathrm{C}$ to $25.82 \pm 2.89 \mathrm{~V}$ at $83.42^{\circ} \mathrm{C}$. Similarly, it is clear that the baseline current at voltages lower than $\mathrm{V}_{b r}$ also increases with temperature and that the shape of the IV curve above $\mathrm{V}_{b r}$ decreases in linearity. This altered shape is further pronounced once the temperature is pushed beyond the rated maximum of $85^{\circ} \mathrm{C}$ and it is no longer a simple matter to determine the breakdown voltage.

If we extract the breakdown voltage from the curves shown in Figure 5 , as well as the remainder of the dataset, its response as a function of temperature becomes clear and can be seen in Figure 6 The full dataset collated in Figure 6 is fitted to a straight line which establishes a tem- perature dependency of $17.7 \pm 0.9 \mathrm{mV} /{ }^{\circ} \mathrm{C}$, close to the manufacturer's quoted value.

\section{1. $\gamma$ Spectroscopy}

The performance of a SiPM-CsI(Tl) system with respect to temperature was investigated with both a fixed bias voltage and with gain matching, ie. an approximately fixed overvoltage, and data was taken for two radioactive sources, ${ }^{137} \mathrm{Cs}$ and ${ }^{22} \mathrm{Na}$. Where a fixed bias voltage was applied the effect of a rise in temperature increasing the breakdown voltage of the SiPM will cause the gain of the device to decrease due to the reduction in overvoltage.

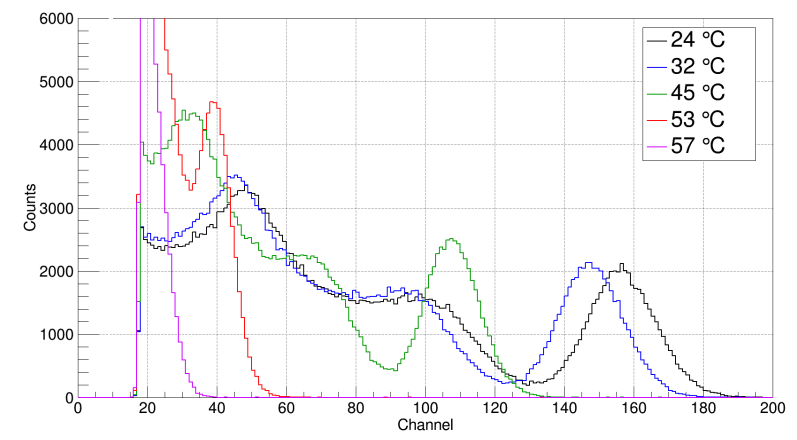

Figure 7: ${ }^{137} \mathrm{Cs} \gamma$-ray spectra with fixed bias voltage of $29.3 \mathrm{~V}$ for different SiPM temperatures. The resolutions of the photopeak (where possible to calculate) are listed in Table 2

The inversely proportional nature of gain to SiPM temperature can be seen clearly in Figure 7 where the voltage was set to $29.3 \mathrm{~V}$. In this case, for the ${ }^{137} \mathrm{Cs}$ dataset, it can be seen that as the temperature of the SiPM increases, the centroid channel number of the $661.7 \mathrm{keV}$ peak decreases, likely a direct result of the reduction of overvoltage. The same trend was seen for the data taken with the ${ }^{22} \mathrm{Na}$ source. Similarly the increased temperature has had a negative effect on the detector $\gamma$ energy resolution, which has been extracted from the data in Figure7 as well as for the equivalent measurements of the ${ }^{22} \mathrm{Na}$ $511 \mathrm{keV}$ peak, which are both displayed in Table 2 .

The potential for gain-matching as a solution to the high temperature environment is shown in Figure 8 
Table 2: Variation of $661.7 \mathrm{keV}$ energy resolution with direct heating of the SiPM.

\begin{tabular}{c|c||c|c}
\hline \multicolumn{2}{c||}{${ }^{137} \mathrm{Cs}-661.7 \mathrm{keV}$} & \multicolumn{2}{c}{${ }^{22} \mathrm{Na}-511 \mathrm{keV}$} \\
Temp. ${ }^{\circ} \mathrm{C}$ & Res. (\%) & Temp. ${ }^{\circ} \mathrm{C}$ & Res. (\%) \\
\hline 24 & $14.8 \pm 0.1$ & 24 & $17.7 \pm 0.1$ \\
32 & $15.3 \pm 0.1$ & 33 & $17.9 \pm 0.1$ \\
45 & $17.3 \pm 0.1$ & 43 & $21.9 \pm 0.1$ \\
53 & $32.7 \pm 0.7$ & 51 & $36.7 \pm 0.2$ \\
57 & $\mathrm{~N} / \mathrm{A}$ & 56 & $\mathrm{~N} / \mathrm{A}$ \\
\hline
\end{tabular}

where the bias voltage has been increased as the temperature increases in an effort to mitigate the effect of the gain reduction due to decreased overvoltage. However, this only has a limited useability with the quoted maximum bias voltage of $31 \mathrm{~V}[43]$ unlikely to provide much assistance for the expected temperatures during TLD readout. An additional confounding factor in this study (although not relevant for implementation in the automated TLD reader) is likely to be the temperature sensitivity of the CsI(Tl) crystal itself[55, 56].

\subsection{Exposure to an External Heat Source}

In order to best approximate the conditions of the TLD readout the soldering iron was taken up to $400^{\circ} \mathrm{C}$, the complimentary work published in [30] has established that $370^{\circ} \mathrm{C}$ is sufficient to fully empty the traps of microsilica glass bead TLDs. Due to the change in heat transfer mechanism, from conductive to radiative, and the new position of the heat source relative to the SiPM, the PT100 no longer provides an accurate measure of the temperature of the SiPM.

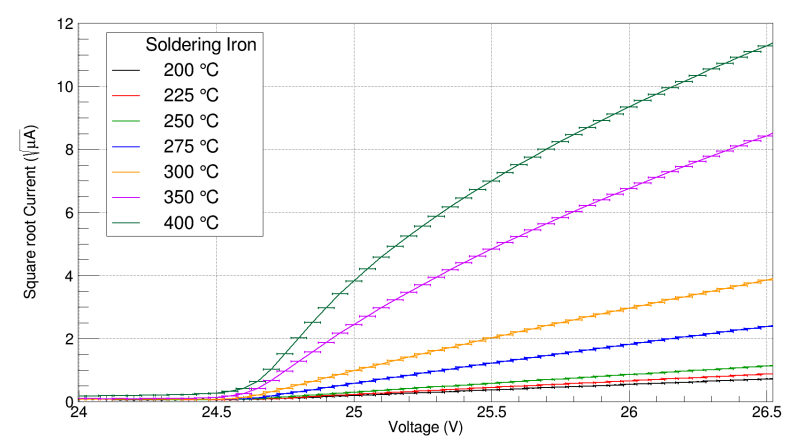

Figure 9: Effect of soldering iron heat source on the breakdown voltage.

The response of the SiPM breakdown voltage and the respective IV curves are shown in Figure 9 for soldering iron temperatures between $200-400^{\circ} \mathrm{C}$. Although it appears that the heat source is indeed following the pattern shown in Figure 5 only the drawn and baseline currents seem to be affected, with the point of semiconductor 


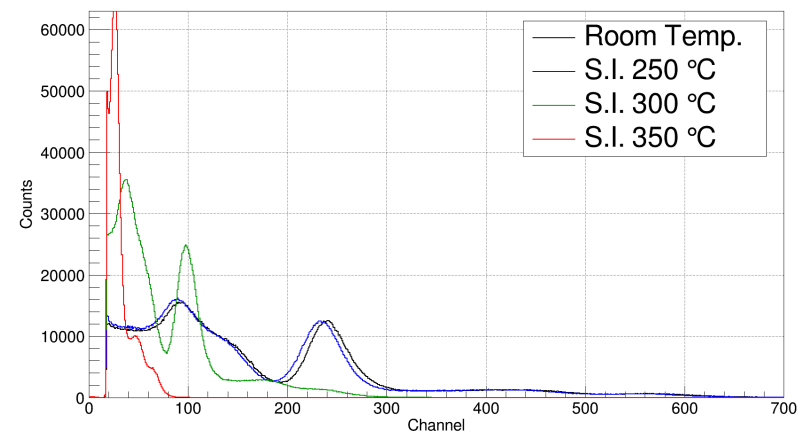

Figure 10: Gamma spectroscopy result for the proximity heating tests at different soldering iron temperatures.

breakdown remaining far more stable than was seen previously.

However, if we now consider the respective $\gamma$-ray spectra displayed in Figure 10, we see an effect greater than that expected if the value of the breakdown voltage was the only factor. The shift in peak centroid from the room temperature measurement to where the heat source was set to $250^{\circ} \mathrm{C}$ is negligible, but once the temperature increases to $300^{\circ} \mathrm{C}$ this channel number more than halves, and then the spectrum becomes unrecogniseable as ${ }^{22} \mathrm{Na}$ at $350^{\circ} \mathrm{C}$. This same trend was also seen with the ${ }^{137} \mathrm{Cs}$ dataset and is present in Figure 9 where the increase in leakage current remains low until the soldering iron is increased to $275-300^{\circ} \mathrm{C}$ indicating the presence of a threshold effect. The $\sim 250^{\circ} \mathrm{C}$ region may be the limit at which the local, ambient conditions can dissipate the soldering iron iduced temperature without the SiPM being affected, and the point at which alternate cooling techniques must be implemented to ensure continued functionality of the system.

\subsection{Active Cooling}

The Peltier module's suitability as a means of cooling the SiPM was tested at an external temperature source of $300^{\circ} \mathrm{C}$ and the respective IV curves for these are depicted in Figure 11. There is a noticeable shift in breakdown voltage once the Peltier is switched on, even at the relatively

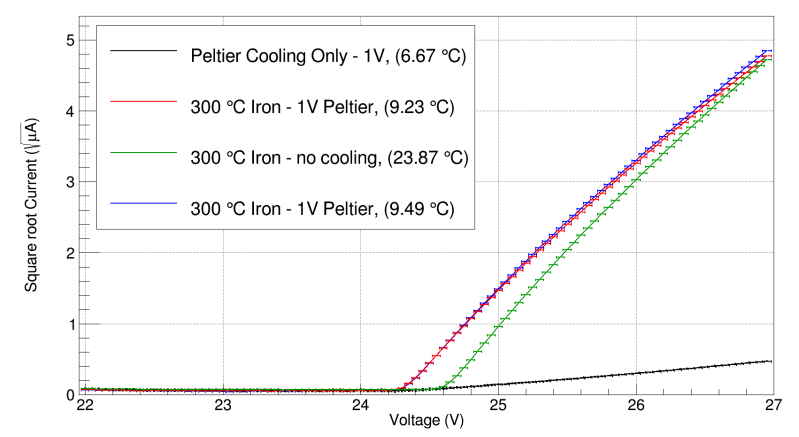

Figure 11: The response of the SiPM breakdown voltage to the active cooling technique. The soldering iron was off in the black data series but otherwise set to $300^{\circ} \mathrm{C}$. The temperature stated within parentheses is that as recorded by the PT100 thermocouple underneath the SiPM evaluation board.

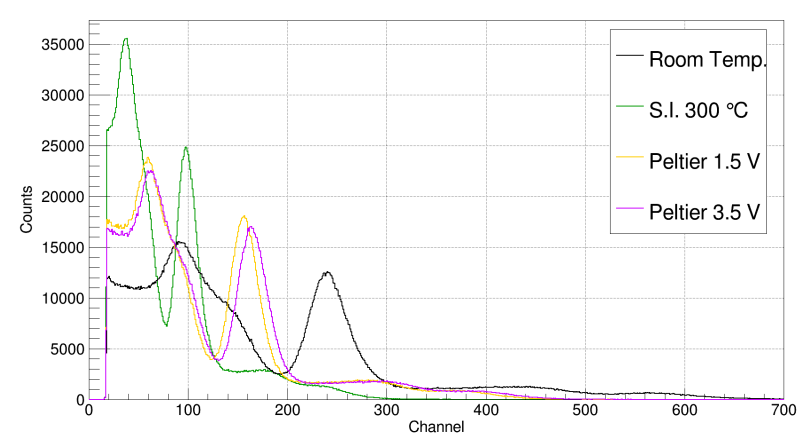

Figure 12: The $\gamma$ spectroscopy response to Peltier active cooling.

low voltage setting of $1 \mathrm{~V}$.

The impact of the active cooling technique, certainly when using only a single Peltier module, is shown to be limited in Figure 12. As was seen previously in Figure 10 the application of a $300^{\circ} \mathrm{C}$ soldering iron in close proximity to the SiPM-Scintillator unit causes a great reduction in the centroid of the photopeak. However, once direct cooling to the SiPM evaluation board is applied by the Peltier Figure 12 shows that this allows for some level of response recovery. Setting the Peltier voltage to $1.5 \mathrm{~V}$ produced a recovery of $\sim 1 / 2$, however, increasing the voltage to near the optimum value at $3.5 \mathrm{~V}$ only produced a minor improvement (for comparison these voltages corresponded to a change in SiPM temperature of approximately 34 and $75^{\circ} \mathrm{C}$ in the direct heating section of this 
work).

\section{Conclusions and Further Work}

Clearly the SiPM is a temperature dependent device, this has been widely reported[36, 57, 58] and confirmed in this work, and potentially has limitations on the suitability of its use where the device itself will be operated at higher temperatures. The performances presented here would suggest that in applications where the SiPM will be exposed to relatively warm conditions during operation it is necessary for some level of temperature compensation to be implemented. However, it is also apparent that the techniques investigated in this work have the potential to fulfil this role.

Additionally, for the intended application in a TLD reader there are a number of further experiments that need to be performed in order to assess the suitability of a SiPM for the photodetection component.

1. Replacement of the soldering iron with TLD heater.

2. Time-integrated light collection as a function of temperature.

3. Application of multi-layer cooling arrays.

4. Performance of alternate cooling techniques.

With the exception of experiment 3 , all of the above suggestions will require construction of a prototype which more closely approximates an end unit. The replacement of the soldering iron with an element such as the kanthal alloy heater described in [28] would enable the real IV curve of the end product to be studied. Similarly, although studying the $\gamma$-ray spectra of a ScintillatorSiPM system as a function of temperature is a standard assessment technique [5, 59], the desired measurement is actually the time-integrated light emitted from the TLD as it is heated, the glow curve. This experiment is clearly the most fundamental to the product but requires a degree of further development work before it can be performed.
The thermoelectric cooling method is limited by a maximum $\Delta \mathrm{T}$ for an individual module. However, this $\Delta \mathrm{T}$ value can be improved through the use of Peltier cascades [60, 61], with multiple modules acting in combination. There are, naturally, diminishing returns through additional modules, and the practicalities (successive cooling stages requiring larger surface area) may make the technique of limited use, but it still remains a possible solution or part thereof.

Finally, it should also be noted that current commercial TLD readers frequently make use of a forced nitrogen flow through the read-out chamber in order to reduce the effect of surface contamination[62]. It is thought the application of this may provide the necessary cooling required for a SiPM to not be exposed to detrimental temperature conditions. This will clearly be a crucial area of investigation for the next stage of this work.

\section{Acknowledgements}

The authors would like to acknowledge the support of SME Innovation Voucher number KN9166N.

\section{References}

[1] M. Caccia, et al., Silicon Photomultiplier Readout of a Scintillating Noble Gas Detector for Homeland Security, in: Proceedings of ANIMMA, 2013.

[2] M. Caccia, Silicon Photomultipliers: Introducing the Digital Age in Low Light Detection, in: Proceedings of MIXDES, 2015.

[3] M. P. Taggart, et al., Development of radiodetection systems towards miniaturised quality control of PET and SPECT radiopharmaceuticals, Lab. Chip 16 (2016) 1605-1616.

[4] A. Pappalardo, et al., Performance evaluation of SiPM's for low threshold gamma detection, Nucl. Phys. B (Proc. Suppl.) 215 (2011) $41-43$.

[5] B. Seitz, N. C. Rivera, A. G. Stewart, Energy Resolution and Temperature Dependance of Ce:GAGG Coupled to $3 \mathrm{~mm} \times 3 \mathrm{~mm}$ Silicon Photomultipliers, IEEE Trans. Nucl. Sci. 63 (2) (2016) 503-508.

[6] I. Sonni, et al., Initial experience with a SiPM-based PET/CT scanner: influence of acquisition time on image quality, EJNMMI Physics 5. 
[7] ET Enterprises, 9102B series data sheet issue 8 (June 2010). URL my.et-enterprises.com/pdf/9102B.pdf

[8] Hamamatsu, Photomultiplier tube: R647, https://www. hamamatsu.com/us/en/R647.html accessed 2018-10-30.

[9] Hamamatsu, Photomultilier tube: R329-02, www.hamamatsu.com/ eu/en/product/type/R329-02/index.html. accessed 2018-10-30.

[10] G. F. Knoll, Radiation, Detection, and Measurement, 3rd Edition, Wiley, 2000.

[11] J. D. Thiessen, et al., Performance Evaluation of SensL SiPM Arrays for High-Resolution PET, in: IEEE Nuclear Science Symposium Conference Record, 2013.

[12] J. Wehner, et al., PET/MRI insert using digital SiPMs: Investigation of MR-compatibility, Nucl. Instr. Meth. A 734 (2014) 116-121.

[13] D. Shug, et al., Initial PET performance evaluation of a preclinical insert for PET/MRI with digital SiPM technology, Phys. Med. Biol. 61 (2016) 2851-2878.

[14] D. A. Bradley, et al., The development of doped radiosensitive glass, in: AIP Conf. Proc., Vol. 909, 2007, pp. 9-18.

[15] C. Bassinet, et al., Small fields output factors measurements and correction factors determination for several detectors for a $\mathrm{Cy}$ berknife and linear accelerators equipped with MicroMLC and circular cones, Med. Phys. 40 (2013) 071725.

[16] M. Teoh, et al., Volumetric modulated arc therapy: a review of current literature and clinical use in practice, Br. J. Radiol. 84 (2011) 967-96.

[17] B. Chang, R. Timmerman, Stereotactic body radiation therapy: a comprehensive review, Am. J. Clin. Oncol. 30 (2007) 637-44.

[18] G. Distefano, et al., A national dosimetry audit for stereotactic ablative radiotherapy in lung, Radiotherapy and Oncology 122 (2017) 406-410.

[19] F. H. Attix, Introduction to Radiological Physics and Radiation Dosimetry, Wiley, 2007.

[20] A. E. Nahum, Perturbation effects in dosimetry: Part I. Kilovoltage x-rays and electrons, Phys. Med. Biol. 41 (1996) 1531.

[21] T. C. Zhu, Small Field: dosimetry in electron disequilibrium region, J. Phys. Conf. Ser. 250 (2010) 012056.

[22] S. Jafari, et al., Low-cost commercial glass beads as dosimeters in radiotherapy, Radiat. Phys. Chem. 97 (2014) 95-101.

[23] R. Cameron, Thermoluminescent Dosimetry, The University of Wisconsin Press, 1968.

[24] J. C. McDonald, Calibration measurements and standards for radiation protection dosimetry, Radiat. Prot. Dosim. 109 (2004) 317321.

[25] Y.S. Horowitz, D. Yossian, Computerised Glow Curve Deconvolution: Application to Thermoluminescence Dosimetry, Radiat. Prot. Dosim. 60 (1995) 1-114.

[26] M. Ranogajec-Komor, F. Muhiy-Ed-Din, D. Milkovic, B. Veckic, Thermoluminesence Characteristics of Various Detectors for X-ray
Diagnostic Measurements, Radiat. Prot. Dosim. 47 (1993) 529-534.

[27] M. Šimpraga, M. Ranogajec-Komor, V. Butkovic, D. Capak, Use of an animal model for the determination of radiation dose distribution in X-ray diagnostics, Veterinarski Arhiv 69 (1999) 289-298.

[28] DTU Nutech, Guide to "The Risø TL/OSL Reader"|(2015).

URL https://www.nutech.dtu.dk/english/ products-and-services/radiation-instruments/tl_osl_ reader/manuals

[29] D. Wróbel, et al., Characterization of the Risø TL/OSL DA-20 reader for application in TL dosimetry, Radiation Measurements 74 (2015) 1-5.

[30] K. Ley, et al., Investigations of thermoluminescent silica beads of different manufacturers, Radiat. Phys. Chem. 155 (2019) 178-183.

[31] G. D. Glennie, A comparison of TLD dosimeters: LiF:Mg,Ti and LiF:Mg,Cu,P for measurement of radiation therapy doses, Ph.D. thesis, University of Virginia (2003).

[32] S. D. S. Fernández, et al., Thermoluminescent characteristics of $\mathrm{LiF}: \mathrm{Mg}, \mathrm{Cu}, \mathrm{P}$ and $\mathrm{CaSO}_{4}$ :Dy for low dose measurement, Applied Radiation and Isotopes 111 (2016) 50-55.

[33] L. Duggan, et al., Suitability of $\mathrm{LiF}: \mathrm{Mg}, \mathrm{Cu}, \mathrm{P}$ and $\mathrm{Al}_{2} \mathrm{O}_{3}: \mathrm{C}$ for low dose measurements in medical imaging, Radiation Protection Dosimetry 85 (1-4) (1999) 425-428.

[34] A. Romanyukha, M. D. Grypp, G. R. Fairchild, A. S. Williams, Performance comparison of OSLD $\left(\mathrm{Al}_{2} \mathrm{O}_{3}: \mathrm{C}\right)$ and TLD $(\mathrm{LiF}: \mathrm{Mg}, \mathrm{Cu}, \mathrm{P})$ in accreditation proficiency testing, Radiation Measurements 93 (2016) 7-12.

[35] C. Piemonte, et al., Characterization of the First Prototypes of Silicon Photomultiplier Fabricated at ITC-irst, IEEE Trans. Nucl. Sci. 54 (1) (2007) 236-244.

[36] M. Ramilli, Characterization of SiPM: temperature dependencies, in: IEEE Nuclear Science Symposium Conference Record, 2008.

[37] V. Puill, et al., Single photoelectron timing resolution of SiPM as a function of the bias voltage, the wavelength and the temperature, Nucl. Instr. Meth. A 695 (2012) 354-358.

[38] W. Hunter, R. Miyaoka, L. MacDonald, T. Lewellen, Measured Temperature Dependence of Scintillation Camera Signals Read Out by Geiger-Müller Avalanche Photodiodes, in: IEEE Nuclear Science Symposium Conference Record, 2009.

[39] SensL Technologies Ltd., How to Evaluate and Compare Silicon Photomultiplier Sensors, rev. 1.1 (October 2015) [cited January 2019].

URL sensl.com/downloads/ds/WP-Evaluate_and_Compare_ SiPM.pdf

[40] A. Ulyanov, et al., Localisation of gamma-ray interaction points in thick monolithic $\mathrm{CeBr}_{3}$ and $\mathrm{LaBr}_{3}$ :Ce scintillators, Nucl. Instr. Meth. A 844 (2017) 81-89.

[41] R. Raylman, A. Stolin, Immersion cooling of silicon photomultipliers (SiPM) for nuclear medicine imaging applications, Radiation 
Measurements 85 (2016) 111-115.

[42] A. Sokolov, V. Gostillo, A. Loupilov, V. Zalinkevich, Performance Improvement of $\mathrm{Si}(\mathrm{Li})$ Peltier Cooler Detectors, IEEE Trans. Nucl. Sci. 49 (5) (2002) 2427-2430.

[43] SensL Technologies Ltd., J-series: High PDE and Timing Resolution, TSV Package Datasheet rev. 3.1 (2017).

URL sensl.com/downloads/ds/DS-MicroJseries.pdf

[44] S. L. Bugby, L. K. Jambi, J. E. Lees, A comparison of CsI:Tl and GOS in a scintillator-CCD detector for nuclear medicine imaging, JINST 11.

[45] Y. Wang, et al., High-DQE EPIDs based on thick, segmented BGO and CsI:Tl scintillators: Performance evaluation at extremely low dose, Med. Phys. 36 (2009) 5707-5718.

[46] W. Nagarkar, et al., New design of a structured CsI(Tl) screen for digital mammography, in: Medical Imaging 2003: Physics of Medical Imaging, Vol. 5030, 2003, pp. 541-6.

[47] H. Sabet, L. Blackberg, D. Uzun-Ozsahin, G. El-Fakhri, Novel laserprocessed CsI(Tl) detector for SPECT, Med. Phys. 43 (2016) 26302638.

[48] A. S. Hoover, et al., Gamma-ray imaging with a Si/CsI(Tl) Compton detector, App. Radiat. Iso. 64 (2006) 1648-1654.

[49] M. Bondi, et al., Characterization of a CsI(Tl)-SiPM detector to low energy protons for light dark matter search in a future Beam Dump eXperiment, JINST 12.

[50] G. Cardella, et al., Particle gamma correlations in ${ }^{12} \mathrm{C}$ measured with the $\mathrm{CsI}(\mathrm{Tl})$ based detector array CHIMERA, Nucl. Instr. Meth. A 799 (2015) 64-69.

[51] H. S. Lee, et al., Limits on interactions between weakly interacting massive particles and nucleons obtained with $\mathrm{CsI}(\mathrm{Tl})$ crystal detectors, Physical Review Letters 99 (2007) 091301.

[52] Saint-Gobain Crystals, CsI(Tl), CsI(Na) Cesium Iodide Scintillation Material (2017) [cited January 2019].

URL https://www.crystals.saint-gobain.com/ sites/imdf.crystals.com/files/documents/ csitl-and-na-material-data-sheet.pdf

[53] Ortec, Maestro 7 Multichannel Analyzer MCA Emulation Software, http://www.ortec-online.com/Products-Solutions/ Applications-Software-MAESTRO aspx. accessed August 2018.

[54] M. P. Taggart, C. Payne, P. J. Sellin, Neutron-gamma discrimination via PSD plastic scintillator and SiPMs, J. Phys. Conf. Series 763 (2016) 012007.

[55] H. Grassman, E. Lorenz, H.-G. Moser, Properties of CsI(Tl) - Renaissance of an old scintillation material, Nucl. Instr. Meth. A 228 (1985) 323-326.

[56] J. D. Valentine, et al., Temperature dependence of CsI(Tl) gammaray excited scintillation characteristics, Nucl. Instr. Meth. A 325 (1993) 147-157.

[57] N. Dinu, A. Nagai, A. Para, Breakdown voltage and triggering probability of SiPM from IV curves at different temperatures, Nucl. Instr. Meth. A 845 (2017) 64-68.

[58] M. Biroth, P. Achenbach, W. Lauth, A. Thomas, Modeling and Characterization of SiPM Parameters at Temperatures Between 95 K and 300 K, IEEE Trans. Nucl. Sci. 64 (2017) 1619-1624.

[59] N. Dinar, D. Celeste, P. Puzo, M. Silari, Characterization of CLYC scintillator coupled with photomultipliers and a large SiPM array, Radiat. Prot. Dosim. 180 (2018) 399-402.

[60] B. J. O'Brien, C. S. Wallace, K. Landecker, Cascading of Peltier Couples for Thermoelectric Cooling, J. App. Phys. 27 (1956) 820-823.

[61] T. Metzger, R. P. Huebener, Modelling and cooling behaviour of Peltier cascades, Cryogenics 39 (1999) 235-239.

[62] S. W. S. McKeever, Thermoluminesence of Solids, Cambridge University Press, 1988. 